Check for updates

Cite this: RSC Adv., 2018, 8, 37965

Received 9th August 2018

Accepted 28th October 2018

DOI: $10.1039 / c 8 r a 06704 j$

rsc.li/rsc-advances

\section{Purification and structural elucidation of chondroitin sulfate/dermatan sulfate from Atlantic bluefin tuna (Thunnus thynnus) skins and their anticoagulant and ACE inhibitory activities}

\author{
Fatma Krichen, ${ }^{a}$ Hajer Bougatef, ${ }^{a}$ Federica Capitani, ${ }^{\mathrm{b}}$ Ikram Ben Amor, ${ }^{\mathrm{c}}$ \\ Imed Koubaa, ${ }^{\text {d }}$ Jalel Gargouri, ${ }^{\mathrm{c}}$ Francesca Maccari, ${ }^{\mathrm{b}}$ Veronica Mantovani, ${ }^{\mathrm{b}}$ \\ Fabio Galeotti, ${ }^{\mathrm{b}}$ Nicola Volpi ${ }^{\mathrm{b}}$ and Ali Bougatef $\mathbb{D}^{\text {*ae }}$
}

\begin{abstract}
Chondroitin sulfate/dermatan sulfate (CS/DS) was extracted from Atlantic bluefin tuna (Thunnus thynnus) skin (SGAT) and was purified and characterized. SGAT was characterized by acetate cellulose electrophoresis, FTIR spectroscopy, ${ }^{13} \mathrm{C}$ NMR spectroscopy and SAX-HPLC. According to the results obtained for specific chondroitinases (ABC and $A C$ ) and the SAX-HPLC separation of generated unsaturated repeating disaccharides, the polymer was found to contain a disaccharide monosulfated in positions 6 and 4 of GalNAc and disulfated disaccharides in different percentages. These results were confirmed by ${ }^{13} \mathrm{C}$ NMR experiments. The average molecular mass was $24.07 \mathrm{kDa}$, as determined by PAGE analysis. SGAT was evaluated for its in vitro anticoagulant activity via activated partial thromboplastin time, thrombin time and prothrombin time tests. The polymer showed strong inhibitory activity against angiotensin I-converting enzyme $\left(I_{50}=0.25 \mathrm{mg} \mathrm{mL}^{-1}\right)$. Overall, the results suggest that this newly extracted CS/DS can be useful for pharmacological applications.
\end{abstract}

\section{Introduction}

Glycosaminoglycans (GAGs) constitute a group of complex polydisperse carbohydrates that play important roles in numerous biological processes., ${ }^{1,2}$ GAGs are linear polysaccharide chains composed of repeating disaccharide units, each consisting of a hexosamine ( $N$-acetylgalactosamine or $N$ acetylglucosamine) and a uronic acid (D-glucuronic acid or $\mathrm{L}^{-}$ iduronic acid) or galactose, which can be differently sulfated depending on the GAG family. ${ }^{3}$ There are many types of GAGs, and they are generally divided into four groups: (1) hyaluronic acid or hyaluronan; (2) keratan sulfate; (3) chondroitin sulfate (CS)/dermatan sulfate (DS); and (4) heparan sulfate (HS)/ heparin $(\mathrm{H})$.

These anionic polymers are widespread in nature, occurring in a great variety of organisms, such as vertebrates and terrestrial or marine invertebrates. The extraction of GAGs from

${ }^{a}$ Laboratory of Plant Improvement and Valorization of Agroressources, National School of Engineering of Sfax (ENIS), University of Sfax, Sfax 3038, Tunisia. E-mail: ali. bougatef@isbs.usf.tn; Fax: +216 74674 364; Tel: +216 74674354

${ }^{b}$ Department of Life Sciences, University of Modena and Reggio Emilia, Modena, Italy

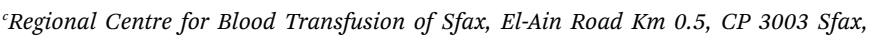
Tunisia

${ }^{d}$ Faculty of Science of Sfax, Laboratory of Chemistry of Natural Products, University of Sfax, Tunisia

${ }^{e}$ High Institute of Biotechnology of Sfax, BP 1175, 3038, Sfax, Tunisia marine invertebrates has been well established; however, their relative amounts, molecular masses and sulfation vary. These marine animal sources include Norway lobster shell, ${ }^{4}$ grey triggerfish and smooth hound skins, ${ }^{5}$ bony fishes, ${ }^{6}$ sturgeon cartilage, ${ }^{7,8}$ ray skin ${ }^{9}$ and shark cartilage. ${ }^{10}$

Sulfated GAGs are extremely diverse in their molecular weights and degrees and positions of sulfation, among other properties. In particular, CS/DS isolated from the skin of marine vertebrates such as corb, grey triggerfish, smooth hound and ray have variable structures and disaccharide compositions. ${ }^{11-13}$ Indeed, CS/DS isolated from the skin of the corb and smooth hound is predominately composed of monosulfated disaccharides bearing sulfate groups at position C-4 of $\mathrm{N}$-acetygalactosamine and disulfated disaccharides bearing esterified sulfate groups at position C-2 of uronic acid and position C-4 of $\mathrm{N}$ acetygalactosamine. ${ }^{11,12}$ However, DS from Raja clavata ray skin has low sulfate content and is composed of non-sulfated and monosulfated disaccharides bearing sulfate groups at positions C-4 or C-6 of $N$-acetyl galactosamine (GalNAc). ${ }^{14}$ Moreover, GAGs possess various molecular masses and polydispersities depending on the source. In this context, CS isolated from 'terrestrial' origins, such as bovine, porcine and avian cartilage, generally has molecular weight values ranging from 13 to 26 $\mathrm{kDa}{ }^{15,16}$ In contrast, CS/DS from fish skin has molecular mass values between 15.46 and $41.72 \mathrm{kDa}^{.11,12}$ In the same context, DS isolated from the skin of the rays Raja clavata and Raja radula 
has molecular masses of $31.2 \mathrm{kDa}$ and $33 \mathrm{kDa}$, respectively. ${ }^{\mathbf{1 3 , 1 7}}$ According to their specific structures, GAGs are involved in various biological processes, such as antihypertensive, ${ }^{18}$ anticoagulant and antithrombotic, ${ }^{19,20}$ anti-inflammatory, ${ }^{21}$ cell proliferation, cell differentiation, cancer metastasis, nerve regeneration, and differentiation of stem cells through intermolecular interactions with different functional proteins.

Anticoagulant activity is among the most widely studied properties of GAGs. Anticoagulants are extensively used in the pharmaceutical field for treating major deadly diseases such as stroke and myocardial infarction. In the treatment of these conditions, medicines with antithrombotic, anticoagulant and anti-platelet activities are mainly used. This is because they inhibit enzymes of the coagulation pathway in the blood circulation system or inhibit the activation and aggregation of platelets. Actually, anticoagulants are derived from mammalian animal sources, in particular $H$. Hence, there is always a threat of various mammalian animal diseases which can affect human health, such as mad cow disease. ${ }^{22}$ In order to develop and extract anticoagulants from safer sources, seafood processing by-products are considered to be a good choice.

Furthermore, hypertension is a major chronic disease which affects $30 \%$ of the adult population worldwide. ${ }^{23}$ It is well known that angiotensin I-converting enzyme (ACE) plays an important role in the fluid and salt balance in mammals as well as the renin-angiotensin and the kallikrein-kinin systems for the regulation of blood pressure. ${ }^{24}$ However, ACE catalyzes the conversion of angiotensin I (an inactive decapeptide) into angiotensin II by cleaving a dipeptide from its C-terminus. Synthetic ACE inhibitors have side effects that include skin rashes, allergic reactions, cough, headache and renal impairment. ${ }^{25}$ Therefore, the replacement of synthetic ACE inhibitors by natural ones can have benefits in terms of health implications and functionality in food systems.

The present work was carried out to characterize and to assess the in vitro anticoagulant and antihypertensive activities of sulfated glycosaminoglycan extracted from the skin of Atlantic bluefin tuna (Thunnus thynnus) (SGAT).

\section{Materials and methods}

\subsection{Reagents}

The chemicals and solvents used in the present study were purchased at analytical grade or the highest level of purity available. Alcalase ${ }^{\circledR} 2.4 \mathrm{~L}$ serine-protease from Bacillus licheniformis was obtained from Novozymes ${ }^{\circledR}$ (Bagsvaerd, Denmark). GAGs standard, CS from bovine trachea, DS from porcine intestinal mucosa, and HS (heparan sulfate) from bovine kidney were obtained from Sigma-Aldrich (St. Louis, MO, USA). Chondroitinase $\mathrm{ABC}$ from Proteus vulgaris (EC 4.2.2.4), specific activity of 0.5 to 2 units per $\mathrm{mg}$, was obtained from SigmaAldrich. Chondroitinase AC from Flavobacterium heparinum (EC 4.2.2.5), specific activity of 0.5 to 2 units per $\mathrm{mg}$, was obtained from Sigma-Aldrich. Unsaturated chondro/dermato disaccharides $[\Delta$ DioS $(\Delta \mathrm{UA}-[1 \rightarrow 3]$-GalNAc), $\Delta$ Di4S $(\Delta \mathrm{UA}-[1 \rightarrow$ 3]-GalNAc-4s), $\Delta$ Di6S $(\Delta \mathrm{UA}-[1 \rightarrow 3]$-GalNAc-6S), $\Delta$ Di2S( $\Delta$ UA-2S$[1 \rightarrow 3]$-GalNAc), $\Delta$ Di2,4diS $(\Delta$ Di-dis B, $\Delta$ UA-2S-[1 $\rightarrow 3]$-GalNAc-
4S), $\Delta$ Di2,6diS ( $\Delta$ Di-diS D, $\Delta$ UA-2S-[1 $\rightarrow 3]$ GalNAc-6S), $\Delta$ di4,6dis $(\Delta$ Di-dis E, $\Delta \mathrm{UA}-[1 \rightarrow 3]$-GalNAc-4,6diS), and $\Delta \mathrm{Di} 2,4,6$ triS $(\Delta$ Ditris, $\Delta$ UA-2S-[1 $\rightarrow 3]$-GalNAc-4S,6S)] were obtained from Seikagaku Corporation (Tokyo City, Japan) and Sigma-Aldrich. Diethylaminoethyl (DEAE)-cellulose was purchased from Pharmacia (Uppsala, Sweden). Angiotensin I-converting enzyme from rabbit lung and the ACE synthetic substrate hippuryl-Lhistidyl-L-leucine (HHL) were purchased from Sigma Chemical Co. (St. Louis, MO, USA). Heparin sodium Choay was obtained from Sanofi-Aventis (France).

\subsection{Preparation of fish skin}

Fish by-products were obtained from a local fish market in Sfax, Tunisia. The samples were packed in polyethylene bags, placed in ice with a sample/ice ratio of approximately $1: 3(\mathrm{w} / \mathrm{w})$, and transported to the research laboratory within $30 \mathrm{~min}$. Upon arrival, the samples were washed twice with water and separated. Only the outer skin of the fish was collected; the skin was then stored in sealed plastic bags at $-20^{\circ} \mathrm{C}$ until further use for the extraction and analysis of GAGs.

\subsection{Enzymatic extraction of sulfated glycosaminoglycans}

GAGs were extracted according to a slightly modified version of the method described by Ben Mansour et al. ${ }^{9}$ In brief, the fish skins were cut into small pieces and homogenized using a Moulinex R62 homogenizer (Organotechnie, Courneuve, France). A $5 \mathrm{~g}$ amount of sample was dissolved in $250 \mathrm{~mL}$ sodium acetate $(0.1 \mathrm{M})$, EDTA $(5 \mathrm{mM})$, and cysteine $(5 \mathrm{mM})$ at pH 6. Alacalase ${ }^{\circledR}$ was added, and the mixture was maintained for $24 \mathrm{~h}$ at $50{ }^{\circ} \mathrm{C}$. The mixture was then allowed to cool to room temperature and then filtered. The residue was washed with distilled water and filtered again. The filtrates were mixed, and the polysaccharides were precipitated with cetylpyridinium chloride $10 \%(\mathrm{w} / \mathrm{v})$. The mixture was maintained for $24 \mathrm{~h}$ at room temperature and centrifuged for $30 \mathrm{~min}$ at $5000 \mathrm{RPM}$ and $4{ }^{\circ} \mathrm{C}$ using a refrigerated centrifuge (Hettich Zentrifugen, ROTINA 380R, Germany). The pellet was washed with cetylpyridinium chloride $0.05 \%(\mathrm{w} / \mathrm{v})$ and then dissolved in $200 \mathrm{~mL}$ $\mathrm{NaCl}$ solution in ethanol $(100: 15, \mathrm{v} / \mathrm{v}) .700 \mathrm{~mL}$ of ethanol was added. The polysaccharide-containing solution was maintained for $24 \mathrm{~h}$ at $4{ }^{\circ} \mathrm{C}$ and then centrifuged for $30 \mathrm{~min}$ at $5000 \mathrm{RPM}$ and $4{ }^{\circ} \mathrm{C}$. The pellet was washed twice with $80 \%$ ethanol and then once with absolute ethanol. After that, the pellet was redissolved in deionised water and lyophilized in a freeze dryer (Martin Christ, Alpha 1-2 LDplus, Germany).

\subsection{Purification of fish skin GAGs}

The lyophilized sulfated GAGs were suspended in distilled water and then fractionated by anion-exchange chromatography on a DEAE-cellulose column $(2 \mathrm{~cm} \times 6 \mathrm{~cm})$ equilibrated with $50 \mathrm{mM} \mathrm{NaCl}$. The GAGs were eluted with a linear gradient of $\mathrm{NaCl}$ from $50 \mathrm{mM}$ to $2 \mathrm{M}$ from 0 to $150 \mathrm{~min}$ at a flow of 1 $\mathrm{mL} \mathrm{min}^{-1}$. Two volumes of ethanol were added to the collected fractions corresponding to single species of GAGs evaluated by uronic acid assay, ${ }^{26}$ and the GAGs were precipitated at $4{ }^{\circ} \mathrm{C}$. 
After centrifugation at $10000 \mathrm{~g}$ for $10 \mathrm{~min}$, the pellet was dried at $50{ }^{\circ} \mathrm{C}$ and solubilized in distilled water.

\subsection{Chemical composition analysis}

Total carbohydrates were determined by the phenol-sulphuric acid method. ${ }^{27}$ Total uronic acid content was quantified colorimetrically according to the method described by Cesaretti $e t$ $a l .{ }^{26}$ The sulfate content in the GAGs was assessed by liquid-ion chromatography (HPLC) on a Metrohm chromatograph equipped with CI SUPER-SEP columns using phthalic acid and acetonitrile as eluents. The test precision of the instrument was about $\pm 2 \%$. All measurements were performed in triplicate.

\subsection{Determination of colour}

The GAGs were placed between two steel dishes with a hole $5.7 \mathrm{~cm}$ in diameter. The colour of the film was determined with a tristimulus colorimeter (CHROMA METER CR-400/410. KONICA MINOLTA, Japan) using the CIE Lab scale $\left(C / 2^{\circ}\right)$, where $L^{*}, a^{*}$ and $b^{*}$ are the parameters that measure lightness, redness and yellowness, respectively. A standard white plate was used as a reference. The results were the average of three measurements taken at ambient temperature at different points on the samples.

\subsection{Cellulose acetate electrophoresis}

The cellulose acetate electrophoresis was performed as follows: $3.5 \mu \mathrm{g}$ of the GAG standard solution containing DS, CS and HS and $3.5 \mu \mathrm{g}$ of the purified sulfated GAGs from fish skins were placed at the origin $(10 \mathrm{~mm}$ from the cathode side) of a cellulose acetate strip (Sartorius). Electrophoresis was carried out in Znacetate $0.1 \mathrm{M} \mathrm{pH} 6$ buffer run at $60 \mathrm{~V}$ at room temperature for $2 \mathrm{~h}$. After electrophoresis, the cellulose acetate strip was stained with alcian blue. ${ }^{28}$

\subsection{UV spectroscopy analysis}

The CS/DS solution was prepared by suspending the sample in distilled water to a concentration of $1.0 \mathrm{mg} \mathrm{mL} \mathrm{m}^{-1}$ for UV measurement in the wavelength range of 190 to $700 \mathrm{~nm}$. Distilled water was used as a control in all processes.

\subsection{CS/DS molecular mass determination}

The molecular mass of CS/DS obtained from Atlantic bluefin tuna skins (SGAT) was determined by PAGE according to Edens et al. ${ }^{29} 15 \mu \mathrm{g}$ of the purified CS/DS determined by uronic acid assay were layered on the gel. The related calibration curve was constructed using oligosaccharide standards of known molecular mass prepared from CS Buzzega et al. ${ }^{30}$ After a run of $40 \mathrm{~min}$ at $100 \mathrm{~V}$, the gel was stained with toluidine blue $(0.1 \%$ in $1 \%$ acetic acid) for $30 \mathrm{~min}$, followed by destaining in $1 \%$ acetic acid. Molecular mass evaluation was performed by densitometric acquisition of the bands and comparison of their migration times on the calibration curve constructed by plotting the retention times of the standards against the logarithms of their molecular mass values.

\subsection{Infra-red spectroscopic analysis}

The absorption spectra of the samples were obtained using FTIR spectroscopy (Analect Instruments fx-6160). The FTIR spectra of the prepared materials were recorded between 450 and $4000 \mathrm{~cm}^{-1}$ in a NICOET spectrometer. The transmission spectra of the samples were recorded using $\mathrm{KBr}$ pellets containing $0.1 \%$ of sample.

\subsection{NMR analysis}

The ${ }^{13} \mathrm{C}$ NMR spectrum of purified CS/DS was recorded at 298.1 $\mathrm{K}$ on a Bruker ASX300 instrument equipped with a $5 \mathrm{~mm}$ diameter tunable probe using Bruker software. The sample was prepared by dissolving $30 \mathrm{mg}$ in $1 \mathrm{~mL}$ of $\mathrm{D}_{2} \mathrm{O}$ at a high level of deuteration $(99.997 \%)$ to avoid the presence of relatively high water. The final solution was transferred to an NMR tube. The spectra were recorded at temperatures of $25{ }^{\circ} \mathrm{C}$ to $28{ }^{\circ} \mathrm{C}$ at $100.62 \mathrm{MHz}$ unless otherwise specified. ${ }^{13} \mathrm{C}$ chemical shifts $(d$, ppm) are quoted with respect to external sodium 4,4-dimethyl4 -silapentane-1-sulfonate $(0.0 \mathrm{ppm})$.

\subsection{Enzymatic treatments and disaccharide composition evaluation}

After treatment of the purified CS/DS samples with chondroitinase $\mathrm{ABC}$ or $\mathrm{AC}$, the generated unsaturated disaccharides were separated and quantified by anion-exchange (SAX) by means of HPLC equipment from Jasco equipped with a $150 \mathrm{~mm}$ $\times 4.6 \mathrm{~mm}$ Spherisorb 5-SAX stainless-steel column $(5 \mu \mathrm{m}$, trimethylammoniopropyl groups $\mathrm{Si}-\mathrm{CH}_{2}-\mathrm{CH}_{2}-\mathrm{CH}_{2}-\mathrm{N}^{+}\left(\mathrm{CH}_{3}\right)_{3}$ in $\mathrm{Cl}^{-}$form, Phase Separations Limited, Deeside Industrial Park, Deeside Clwyd, U.K.) and detection at $232 \mathrm{~nm}$. Isocratic separation was performed using $50 \mathrm{mM} \mathrm{NaCl}$ at $\mathrm{pH} 4.00$ for $5 \mathrm{~min}$ followed by a linear gradient from 5 to $60 \mathrm{~min}$ of $50 \mathrm{mM} \mathrm{NaCl}$ to $1.2 \mathrm{M} \mathrm{NaCl}$ at $\mathrm{pH} 4.00$ at a flow rate of $1.2 \mathrm{~mL} \mathrm{~min}^{-1}$. Authentic unsaturated standard disaccharides were used for qualitative and quantitative purposes.

\subsection{Anticoagulant activity of sulfated glycosaminoglycans}

The activated partial thromboplastin time (aPTT), thrombin time (TT) and prothrombin time (PT) assays were performed using a semi-automatic line STA (Diagnostica Stago). The samples were dissolved in distilled water. All analyses were performed in triplicate, and mean values were considered.

2.13.1. aPTT assay. For the aPTT assay, $45 \mu \mathrm{L}$ of normal citrated platelet poor plasma (PPP) were mixed with $5 \mu \mathrm{L}$ of purified CS/DS at various concentrations and incubated for $3 \mathrm{~min}$ at $37^{\circ} \mathrm{C}$. Then, $50 \mu \mathrm{L}$ of APTT reagent (CK-PREST) were added, and the mixture was incubated for $3 \mathrm{~min}$ at $37^{\circ} \mathrm{C}$. The clotting time was immediately measured after the addition of $100 \mu \mathrm{L}$ of $25 \mathrm{mM} \mathrm{CaCl}_{2}$. The clotting time is expressed in seconds and as a ratio, where the average value of a normal subject was less than 1.2. The negative and positive controls were established by replacing $5 \mu \mathrm{L}$ of SGAT with distilled water and heparin, respectively.

2.13.2. PT assay. For the PT assay, $5 \mu \mathrm{L}$ of purified CS/DS at various concentrations were incubated with $45 \mu \mathrm{L}$ of PPP for 
$3 \mathrm{~min}$ at $37{ }^{\circ} \mathrm{C}$. The clotting time was determined after the addition of $100 \mu \mathrm{L}$ of Néoplastine ${ }^{\circledR}$ CI (DIAGNOSTICA-STAGO).

2.13.3. TT assay. For the TT assay, volumes of $5 \mu \mathrm{L}$ of purified CS/DSat different concentrations were incubated with $45 \mu \mathrm{L}$ of PPP for $3 \mathrm{~min}$ at $37^{\circ} \mathrm{C}$. The clotting time was recorded after the addition of $100 \mu \mathrm{L}$ of thrombin $(80 \mathrm{NIH})$.

\subsection{Hemolytic activity}

The hemolytic activities of the purified CS/DS were determined by a slightly modified version of the method described by Dathe et $a .^{31}$

\subsection{Angiotensin-I-converting enzyme inhibitory activity}

The ACE inhibitory activity was measured in triplicate as previously described by Nakamura et al. ${ }^{32}$ A sample solution (80 $\mu \mathrm{L}$ ) containing different concentrations ( 0.2 to $\left.0.8 \mathrm{mg} \mathrm{mL}^{-1}\right)$ of purified CS/DS from the skin of Atlantic bluefin tuna (SGAT) was mixed with $200 \mu \mathrm{L}$ of $5 \mathrm{mM}$ HHL and then preincubated for $3 \mathrm{~min}$ at $37^{\circ} \mathrm{C}$. SGAT and HHL were prepared in $100 \mathrm{mM}$ borate buffer ( $\mathrm{pH}$ 8.3) containing $300 \mathrm{mM} \mathrm{NaCl}$. The reactions were then initiated by adding $20 \mu \mathrm{L}$ of $0.1 \mathrm{U} \mathrm{mL}^{-1}$ ACE from rabbit lung prepared in the same buffer. After incubation for $30 \mathrm{~min}$ at $37^{\circ} \mathrm{C}$, the enzyme reactions were stopped by the addition of 250 $\mu \mathrm{L}$ of $0.05 \mathrm{M} \mathrm{HCl}$. The liberated hippuric acid (HA) was extracted with ethyl acetate $(1.7 \mathrm{~mL})$ and then evaporated at $90{ }^{\circ} \mathrm{C}$ for $10 \mathrm{~min}$ by rotary evaporation under reduced pressure (rotary evaporator, Heidolph, Germany). The residue was dissolved in $1 \mathrm{~mL}$ of distilled water, and the absorbance of the extract at $228 \mathrm{~nm}$ was determined using a UV-visible spectrophotometer (UV mini 1240 UV/VIS spectrophotometer, Shimadzu, China). The average value from three determinations at each concentration was used to calculate the ACE inhibition rate as follows:

$$
\text { ACE inhibition }(\%)=\left(\frac{B-A}{B-C}\right) \times 100
$$

where $A$ refers to the absorbance of HA generated in the presence of ACE inhibitor, $B$ is the absorbance of HA generated without ACE inhibitor $(100 \mathrm{mM}$ borate buffer at $\mathrm{pH} 8.3$ was used instead of purified $\mathrm{CS} / \mathrm{DS}$ ), and $C$ is the absorbance of HA generated without ACE (corresponding to HHL autolysis in the course of the enzymatic assay).

The $\mathrm{IC}_{50}$ value, defined as the concentration of purified $\mathrm{CS} /$ $\mathrm{DS}\left(\mathrm{mg} \mathrm{mL}^{-1}\right)$ required to inhibit $50 \%$ of ACE activity, was calculated for each sample using non-linear regression from a plot of the ACE inhibition rate versus the sample concentration.

\subsection{Statistical analysis}

All results were expressed as the mean standard deviation (SD). Data were analyzed using the SPSS statistics program (SPSS 10.0 for Windows, SPSS Inc., Chicago, IL). Statistical differences between sample treatments were determined by one-way analysis of variance (ANOVA), and $p<0.05$ was considered statistically significant.

\section{Results and discussion}

\subsection{Extraction, purification and chemical composition of sulfated glycosaminoglycans}

GAGs were extracted for the first time from the skin of Atlantic bluefin tuna (SGAT). After proteolytic treatment, SGAT was purified on an anion-exchange resin. Based on the wet weight, a sulfated GAGs extraction yield of about $7.78 \%$ was obtained. This yield was similar to those previously reported for skins of grey triggerfish and smooth hound. ${ }^{5}$ The approximate composition of the purified SGAT is shown in Table 1 . The total sugars of SGAT determined by the phenol/sulfuric acid method was about $80.12 \%$. The sugar content was similar to those reported for sulfated polysaccharides from squid $(85.06 \%)^{33}$ and red seaweed $(85.6 \%) .{ }^{34}$ Furthermore, quantitative analyses by the carbazole method showed that SGAT contained 69\% uronic acid. This is similar to the uronic acid amount previously described for CS/DS from grey triggerfish skins (70\%). ${ }^{\mathbf{1 2}}$

The values recorded for the sulfate content in SGAT were $25.43 \pm 1.6 \%$. These values are similar to the sulfate amounts previously described by Ben Mansour et al. ${ }^{35}$ for sulfated GAGs extracted from ray skins (25\%). However, they are higher than those previously described for polysaccharides extracted from grey triggerfish $(8.13 \%)$ and smooth hound $(8.77 \%) .{ }^{5}$ It may therefore be concluded that the amount of sulfate in GAGs depends on their origin, nature of raw material, and type of extraction. Duarte et $a .^{36}$ indicated that the bioactivities of polysaccharides depend on the degree and position of sulfation.

Generally, color influences the overall acceptability of products. In this respect, the analysis revealed that the colour of SGAT was light (80.74). The results also showed that SGAT displayed a relatively high degree of redness $\left(a^{*}=15.71\right)$ but showed low yellowness $\left(b^{*}=1.7\right)$.

\subsection{Purity identification and acetate cellulose electrophoresis}

The ultraviolet-visible spectrum of SGAT in the range of 190 to $700 \mathrm{~nm}$ is presented in Fig. 1A. The SGAT sample showed a stronger absorption peak at 200 to $205 \mathrm{~nm}$, which is characteristic of polysaccharides. However, SGAT had no absorbance at 260 or $280 \mathrm{~nm}$, indicating the absence of nucleic acids and

Table 1 Chemical composition of sulfated glycosaminoglycans purified from Atlantic bluefin tuna skin (SGAT). Values are given as mean \pm SD from triplicate determinations. The physico-chemical composition was calculated based on dry matter

\begin{tabular}{lc}
\hline & SGAT \\
\hline Total sugar (\%) & $80.12 \pm 1.29$ \\
Uronic acid (\%) & $69 \pm 0.14$ \\
Sulfate (\%) & $25.43 \pm 1.6$ \\
Colour & \\
$L^{*}$ & \\
$a^{*}$ & $80.74 \pm 0.3$ \\
$b^{*}$ & $15.71 \pm 0.12$ \\
Yield (\%) & $1.7 \pm 0.06$ \\
\end{tabular}


A

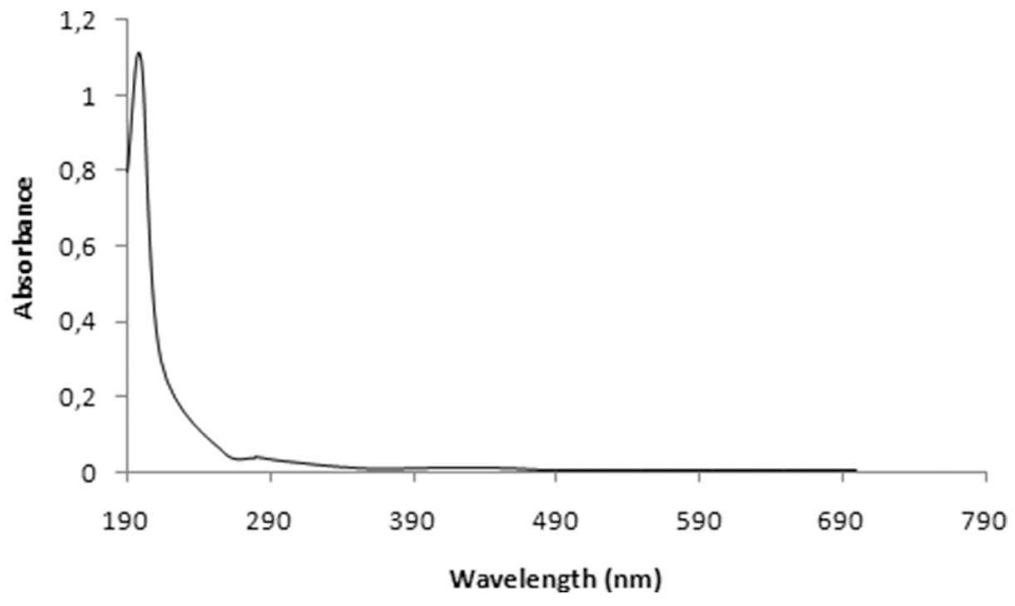

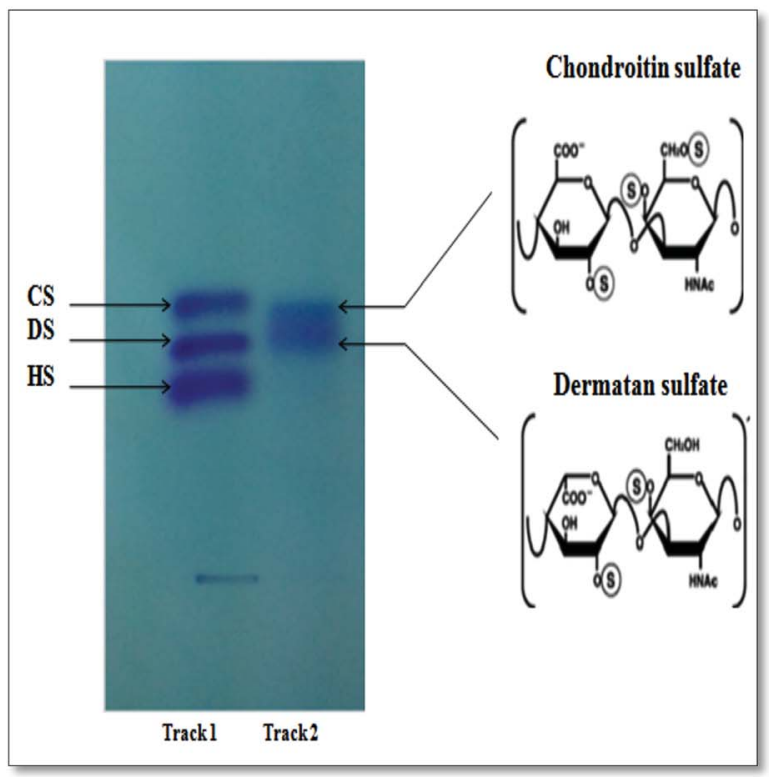

B

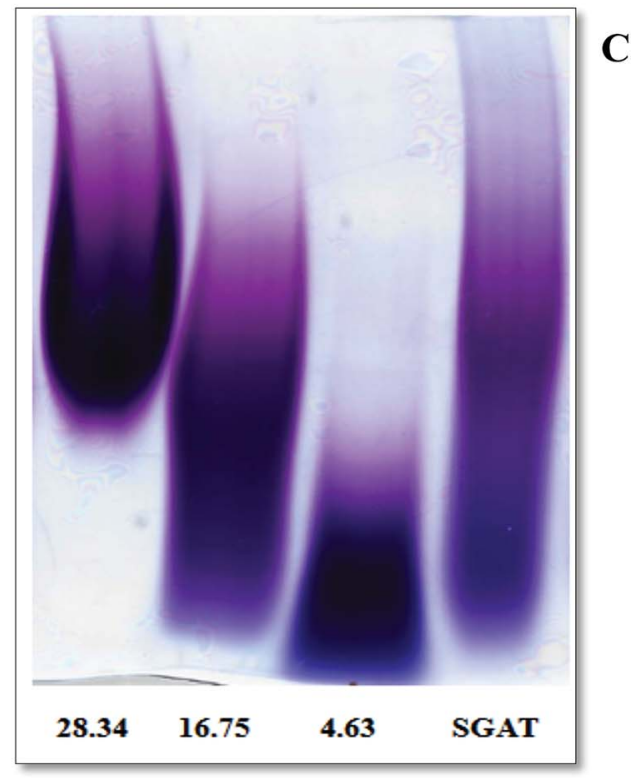

Fig. 1 (A) UV spectrum of the sulfated glycosaminoglycans purified from Atlantic bluefin tuna skin (SGAT) in the wavelength range from 190 to $700 \mathrm{~nm}$ obtained using a UV-vis spectrophotometer. (B) Acetate cellulose electrophoresis of sulfated glycosaminoglycans from Atlantic bluefin tuna skin. Standard GAGs (dermatan sulfate (DS), chondroitin sulfate (CS) and heparan sulfate (HS)) (Track 1), purified GAGs from Atlantic bluefin tuna skin (Track 2). (C) PAGE analysis of CS/DS purified from Atlantic bluefin tuna skin (SGAT). The calibration curve was constructed using standards prepared from chondroitin sulfate with known molecular masses of $4.63 \mathrm{kDa}, 16.75 \mathrm{kDa}$ and $28.34 \mathrm{kDa}$.

proteins. The UV spectrum of sulfated GAGs extracted from Norway lobster showed an extremely weak absorbance at $280 \mathrm{~nm}$, indicating that the sample contains very small amounts of proteins. ${ }^{4}$

The analysis of SGAT by cellulose acetate electrophoresis revealed two single bands visualized with alcian blue and migration; these bands were chondroitin sulfate (CS) and dermatan sulfate (DS) (Fig. 1B). In the same context, Ben Mansour et $a l .{ }^{35}$ reported the isolation of hyaluronic acid and dermatan sulfate from ray skin. Furthermore, Krichen et al. ${ }^{20}$ reported that sulfated GAGs from the skins of smooth hound and grey triggerfish revealed the presence of hyaluronic acid (HA), chondroitin sulfate (CS) and dermatan sulfate (DS).

\subsection{Fish CS/DS molecular mass determination}

It is well known that GAGs from different sources have variable structures and properties. In particular, they can have repeating disaccharides with various numbers and positions of sulfate groups as well as various amounts. Moreover, GAGs possess various molecular masses and polydispersities depending on the source. The PAGE analysis of SGAT was calculated based on a calibration curve of CS fractions of known molecular masses (Fig. 1C). The results showed that SGAT had a molecular mass of around $24.07 \mathrm{kDa}$. This molecular mass was similar to that reported for CS/DS from grey triggerfish $(23.8 \mathrm{kDa}) .{ }^{12}$ In the same context, Chatziioannidis et al. ${ }^{17}$ and Ben Mansour et al. ${ }^{13}$ isolated DS with molecular weights of $31.2 \mathrm{kDa}$ and $33 \mathrm{kDa}$ from skins of the rays Raja clavata and Raja radula, respectively. 
Moreover, Maccari et al. ${ }^{6}$ confirmed that the molecular mass values of CS from bony fishes ranged from 13.46 to $48.68 \mathrm{kDa}$.

\subsection{Infra-red spectroscopic analysis}

FTIR spectroscopy was performed in the 4000 to $450 \mathrm{~cm}^{-1}$ region to further characterize and corroborate the data obtained for SGAT. As shown in Fig. 2A, the spectrum showed strong $\mathrm{OH}$ stretches at $3394 \mathrm{~cm}^{-1}$ and $3150 \mathrm{~cm}^{-1}$. The small band observed at around $2354 \mathrm{~cm}^{-1}$ was attributed to $\mathrm{C}-\mathrm{H}$ stretching. ${ }^{37}$ Furthermore, the presence of uronic acid was evidenced by a strong absorbance band at $1650 \mathrm{~cm}^{-1}(\mathrm{C}=\mathrm{O}$ stretching $){ }^{37}$ The most intense band was observed at $1390 \mathrm{~cm}^{-1}$, corresponding to ester sulfate groups $(\mathrm{S}=\mathrm{O})$. The presence of sulfate (C-O-S) was also confirmed by the absorption at $792 \mathrm{~cm}^{-1} \cdot{ }^{38}$

\subsection{NMR analysis}

The ${ }^{13} \mathrm{C}$ NMR spectrum of SGAT is shown in Fig. 2B. The inspection of the 50 to 70 and 100 to $110 \mathrm{ppm}$ regions revealed a relatively high amount of CS/DS sulfated in position 4 or 6 of the GalNAc. ${ }^{7,39}$ In the ${ }^{13} \mathrm{C}$ NMR spectra, two anomeric carbon signals at 104.1 and $102.08 \mathrm{ppm}$ were attributed to GlcA and GalNAc, respectively. Indeed, the signals at 104.1 and 102.08 were assigned to $\mathrm{C} 1$ of GlcA and to $\mathrm{C} 1$ of GalNAc- $6 \mathrm{SO}_{4}$, respectively, and the signals at 104.03 and 101.11 were assigned to $\mathrm{C} 1$ of GlcA and to $\mathrm{C} 1$ of GalNAc- $4 \mathrm{SO}_{4}$, respectively. These results were similar to those described for sturgeon CS. ${ }^{7}$ Additionally, Mou et al. ${ }^{40}$ reported that the signal at 103.8 can be attributed to $\mathrm{C} 1$ of GlcA in the spectrum of fucosylated CS from sea cucumber. The signals between 50 and $85 \mathrm{ppm}$ were attributed to the chemical shifts of carbon in the glycosylic rings of different monosaccharide residues. Furthermore, the signal at 72.06 was related to $\mathrm{C} 6$ of GalNAc- $6 \mathrm{SO}_{4}$, and an additional signal at 62.5 indicated $\mathrm{C} 6$ of GalNAc- $4 \mathrm{SO}_{4}$; this peak may shift to a higher magnetic field because of the substitution of sugar.

Coppa et al. ${ }^{41}$ reported that the signals at 70.5 and 64.1 in the spectrum of CS from the Italian cheese Parmigiano-Reggiano indicated that $\mathrm{C} 6$ of GalNAc residues was sulfated in positions 6 and 4, respectively. Therefore, the ${ }^{13} \mathrm{C}$ NMR spectrum of SGAT confirmed that this polymer contains a high amount of CS/DS sulfated in positions 4 and 6 of the GalNAc.

\subsection{Enzymatic treatments and disaccharide evaluation of CS/DS}

To characterize the structures of GAGs from fish skins, the purified CS/DS were subjected to treatment with two lyases, chondroitinase $\mathrm{ABC}$ and $\mathrm{AC}$, while the unsaturated disaccharides were analyzed by SAX-HPLC (Fig. 2C).

Chondroitinase $\mathrm{ABC}$ is an endolytic lyase that can totally degrade DS and CS. ${ }^{42}$ GAG lyase enzymes act through an eliminative mechanism to cleave a HexNAc-HexA bond, leaving
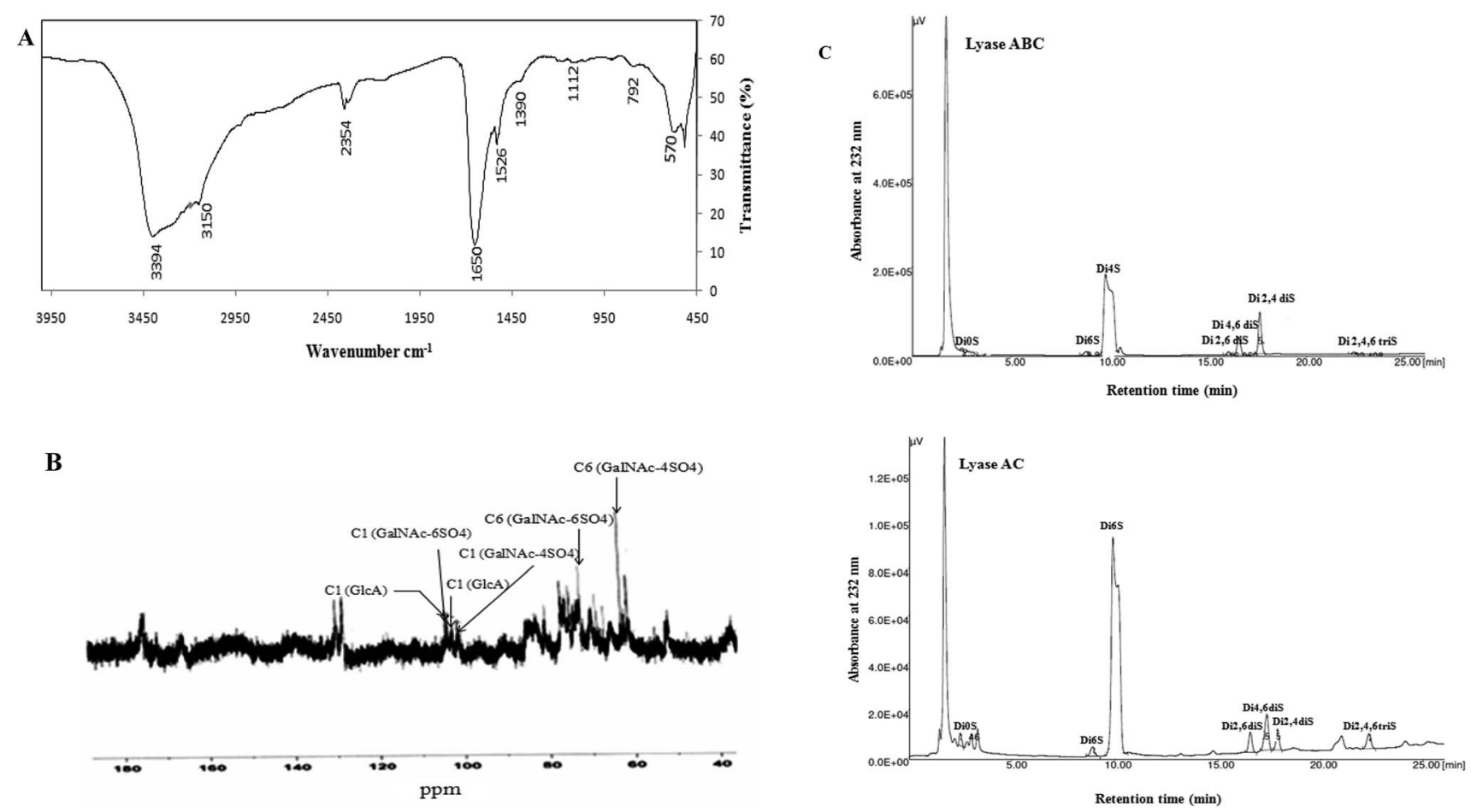

Fig. 2 Structural characterization of SGAT. (A) FT-IR spectrometry of purified CS/DS from Atlantic bluefin tuna skin (SGAT). (B) ${ }^{13} \mathrm{C}$ NMR spectrum of the purified CS/DS from Atlantic bluefin tuna skin. (C) SAX-HPLC separation of the unsaturated disaccharides produced by CS/DS purified from Atlantic bluefin tuna skin and treated with chondroitinase ABC or AC. $\triangle$ DiOS ( $\triangle \cup A-G a l N A c), \Delta$ Di6S $(\Delta \cup A-G a l N A c 6 S), \Delta$ Di4S $(\Delta \cup A-G a l N A c 4 S)$, $\Delta$ Di2,6diS ( $\Delta \cup A 2 S-G a l N A c 6 S) \Delta$ Di4,6diS ( $\Delta \cup A$ GalNAc4, 6diS), $\Delta$ Di2,4diS ( $\Delta$ UA2S-GalNAc4S), $\Delta$ Di2,4,6triS ( $\Delta$ UA2S-GalNAc4,6 diS). The identities of the disaccharide species were determined by co-elution with purified standards (Seikagaku Co./Sigma-Aldrich). All measurements were performed in triplicate. 
a $(4,5)$-unsaturated (termed $\Delta$ unsaturated) HexA residue at the new non-reducing terminus.

As shown in Table 2, chondroitinase ABC produced various unsaturated disaccharides in different percentages from GAGs purified from Atlantic bluefin tuna skin. The percentage of nonsulfated disaccharide DioS of SGAT was trace $(0.671 \%)$, while the percentages of monosulfated disaccharides $\Delta$ Di6S and $\Delta$ Di4S were evaluated to be 2.138 and 74.376 , respectively. Additionally, Krichen et al. ${ }^{12}$ reported that the percentages of monosulfated disaccharides $\Delta$ Di6S and $\Delta$ Di4S recorded in grey triggerfish skins were 18.2 and 59.0, respectively. Furthermore, Maccari et al. ${ }^{6}$ reported that CS extracted from tuna cartilage contained $28.3 \% \Delta \mathrm{Di} 4 \mathrm{~S}$ and $63.2 \% \Delta$ Di6S. In contrast, Hashiguchi et al. ${ }^{43}$ reported that CS extracted from ray fish cartilage contained $61.9 \% \Delta$ Di6S and $27.0 \% \Delta$ Di4S. Interestingly, disulfated disaccharides were observed in SGAT at different rates. SGAT contains a low percentage of disulfated disaccharide $\Delta$ Di2,6dis (1.016\%), while the disulfated disaccharide $\Delta$ Di2,4dis was found in elevated amounts (14.135\%). However, this amount was lower than that previously described by Krichen et $a{ }^{12}{ }^{12}$ for CS/DS from smooth hound skin (20.1\%). Additionally, it is higher than the amount of disaccharide sulfated in positions 2 and 4 in CS/DS from grey triggerfish skin (7.03\%). Furthermore, the results revealed that SGAT had little trisaccharide content $(0.75 \%)$. The presence of disulfated disaccharides produced a high overall charge density of SGAT, which was 1.23. This value is similar to that reported for CS/DS from grey triggerfish and smooth hound skins. ${ }^{12}$
In order to obtain more accurate structure information for SGAT, it was subjected to treatment with chondroitinase AC. This enzyme cleaves GalNAc-GlcA bonds where GalNAc is 4- or 6-O-sulfated but is inactive against GalNAc-IdoA bonds. Therefore, it is only able to cleave chondroitin sulfate. As shown in Fig. 2C and Table 2, chondroitinase AC produced various unsaturated disaccharides in different percentages from SGAT. The analysis by SAX-HPLC chromatography showed that the two chromatograms distinguished disulfated disaccharides in positions 2 and 4 and disulfated disaccharides in positions 2 and 6. By treatment with chondroitinase AC, SGAT was found to be very particular because of the presence inside the polysaccharide backbone of a high percentage of disaccharides with two sulfate groups in positions 2 and 6 of $\mathrm{N}$-acetylgalactosamine $(3.55 \%)$. Furthermore, more of the disulfated disaccharide in positions 2 and 4 (Di2, 4 dis) was produced by chondroitinase ABC than AC (14.13\% and 3.29\%, respectively); this confirms increased concentrations of DS, considering that Di4S and Di2,4diS mainly belong to DS as opposed to the Di6S and Di2,6dis species, which belong to CS. ${ }^{44}$

The percentages of CS and DS were determined by calculating the proportion of the total area of the chromatogram of the AC-samples and the total area of the ABC-samples. In fact, our results suggest that the percentages of CS and DS recovered in SGAT were $39.5 \%$ and $60.5 \%$, respectively.

\subsection{Biological functions of purified CS/DS}

On the basis of the numbers and positions of sulfate on the disaccharides, the biological functions of GAGs are highly

Table 2 Amount, disaccharide composition and charge density of CS/DS purified from Atlantic bluefin tuna skin (SGAT) ${ }^{a}$

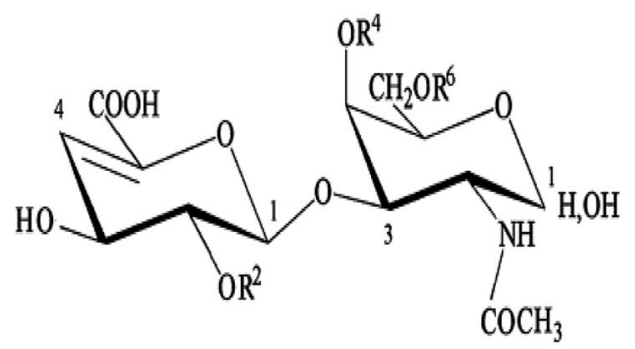

SGAT

Chondroitinase

Chondroitinase AC

$\mathrm{ABC}$

$\Delta$ DioS ( $\triangle$ UA-GalNAc) $(\%)$

$\Delta$ Di6S ( $\triangle$ UA-GalNAc 6S) $(\%)$

$\Delta$ Di4S ( $\triangle$ UA-GalNAc 4 S) $(\%)$

$\Delta$ Di2, 6S ( $\triangle$ UA2S-GalNAc 6S) $(\%)$

$\Delta$ Di4, 6S ( $\triangle$ UA 4S-GalNAc 6S) $(\%)$

$\triangle$ Di2, 4S ( $\triangle$ UA2S-GalNAc 4 S) $(\%)$

$\Delta$ Di2, 4,6 Tris ( $\triangle$ UA2S-GalNAc 4,6 diS) (\%)

Charge density

$\begin{array}{rr}1.566 & 0.671 \\ 2.075 & 2.138 \\ 79.286 & 74.376 \\ 3.555 & 1.016 \\ 7.049 & 6.912 \\ 3.292 & 14.135 \\ 3.180 & 0.753 \\ 1.19 & 1.23\end{array}$

${ }^{a}$ The scheme illustrates the CS/DS unsaturated disaccharides produced by the action of chondroitinase lyases. CS, chondroitin sulfate; DS, dermatan sulfate, $\Delta$ UA, 4,5-unsaturated uronic acid; GalNAc, $N$-acetyl-galactosamine; S, sulfate group. The percentage of each identified disaccharide was determined by purified standards (Seikagaku Co./Sigma-Aldrich) and reported as the weight percentage. Charge density was calculated by considering the number of sulfated groups per disaccharide unit. 
diversified. Accordingly, in the present study, we investigated the anticoagulant and antihypertensive activities of SGAT.

3.7.1. Anticoagulant activity. The effects of SGAT on coagulation time were investigated by the aPTT, PT and TT coagulation assays. These tests were used to distinguish the coagulation phases. aPTT is related to the intrinsic coagulation phase in plasma, PT is related to the extrinsic phase, and TT is associated with the fibrin formation phase in plasma. ${ }^{45,46}$

Numerous reports have revealed that the aPTT test provides information about bleeding risk in preoperative evaluation, monitoring of antithrombotic treatment with heparin and screening of thrombosis risk factors. The anticoagulant activity of SGAT determined with the aPTT test is shown in Fig. 3A. The purified SGAT prolonged the anticoagulant activity in a concentration-dependent manner. Further, the addition of $500 \mu \mathrm{g} \mathrm{mL}{ }^{-1}$ of SGAT in the reaction medium caused a significant $(p<0.05)$ prolongation of the clotting time. The ratio, which reflects the increase in the clotting time of plasma compared to the clotting time of control plasma, is equal to 2.08 at $500 \mu \mathrm{g} \mathrm{mL}{ }^{-1}$. In this context, Sayari et al. ${ }^{4}$ reported that sulfated GAGs from Norway lobster shell prolonged anticoagulant activity 3 times more than the control at a concentration of $100 \mu \mathrm{g} \mathrm{mL}^{-1}$. Furthermore, Ben Mansour et al. ${ }^{9}$ reported concentration-dependent anticoagulant activity as measured by aPTT which was 2 to 3 -fold lower than that of heparin for the skin of the ray Raja radula. The anticoagulant activity of SGAT, revealed by aPTT assay prolongation, was expected because sulfate groups are necessary to provide anticoagulant effects of polysaccharides. These are not only dependent on the sulfate content but also on the position of the sulfate groups and the molecular weight. ${ }^{47,48}$ Previous studies have indicated that the aPTT assay results of the low molecular weight fraction of GAGs from sea cucumber $\left(M_{\mathrm{w}}=24.755 \mathrm{kDa}\right)$ are similar to those of heparin, with significantly fewer side effects. ${ }^{48}$ In the present work, the in vitro anticoagulant activity of SGAT showed relationships with the concentration, the position and amount of sulfate groups and the molecular mass $(\mathrm{MM}=24.07 \mathrm{kDa})$.

Furthermore, it is highly important to compare the effects of SGAT and commercial heparin used as a positive control on clotting times. Heparin caused a significant prolongation of aPTT, and its clotting times were higher than those of SGAT.
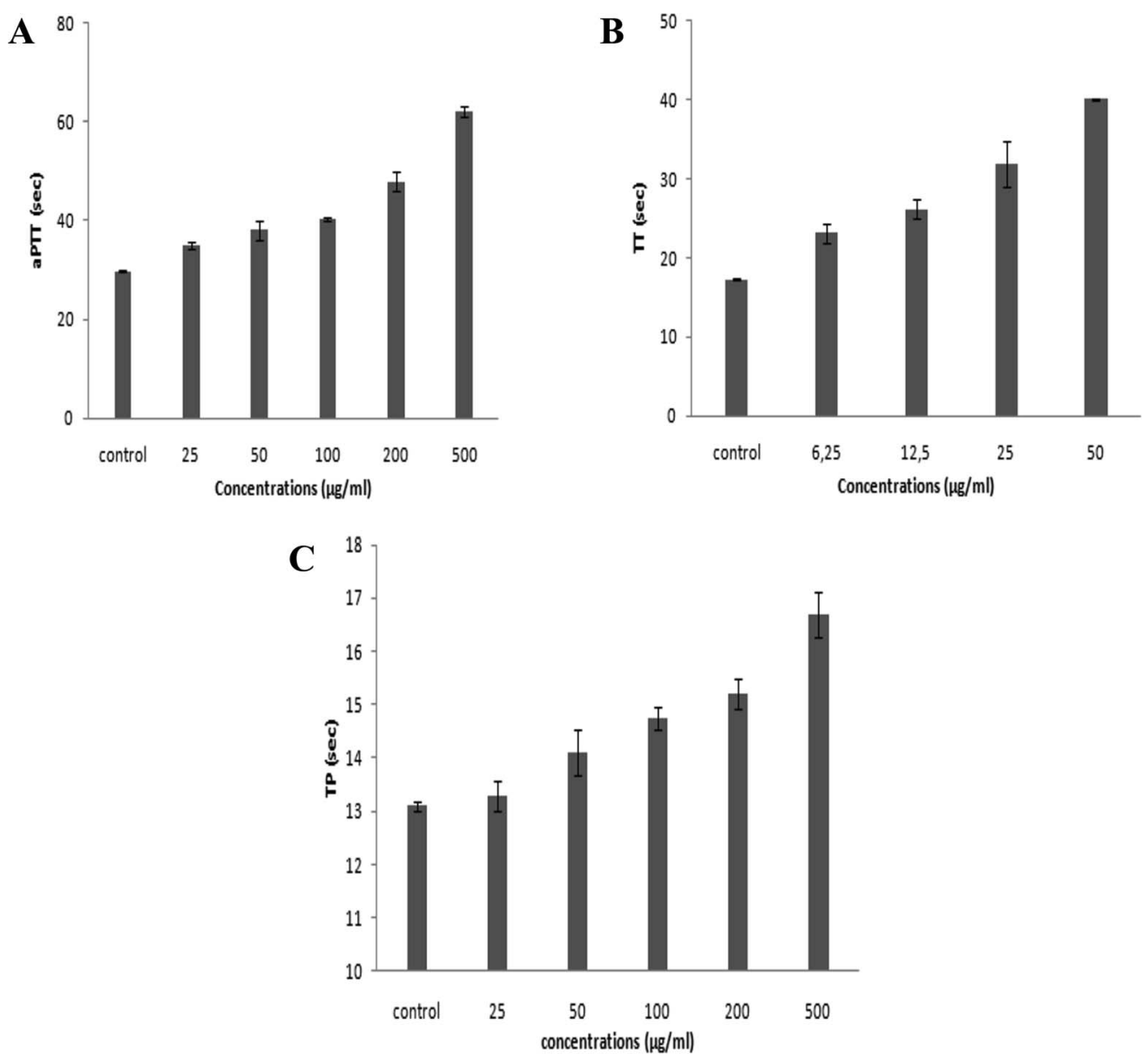

Fig. 3 (A) Anticoagulant activity of the purified CS/DS from Atlantic bluefin tuna skin (SGAT) at different concentrations evaluated by measurement of the activated partial thromboplastin time (aPTT). (B) Anticoagulant activity of SGAT at different concentrations evaluated by measurement of the thrombin time (TT). (C) Anticoagulant activity of SGAT at different concentrations evaluated by measurement of the prothrombin time (PT). All results are expressed as mean $\pm \mathrm{SD}(n=3)$ and all measurements were performed in triplicate. 
Indeed, the use of $7 \mu \mathrm{g} \mathrm{mL} \mathrm{m}^{-1}$ of heparin prolonged the coagulation time, which reached a value greater than $120 \mathrm{~s}$. This clotting time value indicates that heparin can induce high risk of bleeding. As reported in Table 3, the aPTT anticoagulant activity of SGAT was evaluated using a parallel standard curve based on the International Heparin Standard. The aPTT anticoagulant activity of SGAT required $44.5 \mathrm{IU} \mathrm{mg}^{-1}$ at $500 \mu \mathrm{g}$ $\mathrm{mL}^{-1}$. These obtained results were lower compared to the anticoagulant effect of sulfated GAGs from A. pleuronectus. ${ }^{49}$

The anticoagulant effect of purified CS/DS was also evaluated via TT assay. This test is a measure of the conversion of fibrinogen to fibrin by high doses of thrombin, and an increase in TT suggests either thrombin inhibition or impaired fibrin polymerization. ${ }^{50}$ The TT results of SGAT at different concentrations are presented in Fig. 3B, which demonstrates that TT was prolonged in a concentration-dependent manner. Interestingly, SGAT significatively prolonged TT about $2.33(40.1 \mathrm{~s} \pm$ $0.14 \mathrm{~s}$ ) times more than the control at a concentration of $50 \mu \mathrm{g}$ $\mathrm{mL}^{-1}$. In this respect, Yang et al. ${ }^{48}$ studied the anticoagulant activity of GAGs from sea cucumber (Apostichopus japonicas) using the TT assay; they reported that the activities of GAGs were slightly higher than that of heparin at similar concentrations. Furthermore, the TT of SGAT was more prolonged than that previously described for the polysaccharides from Gentiana scabra Bunge roots ( 1.1 fold at the concentration of $1.5 \mathrm{mg}$ $\left.\mathrm{mL}^{-1}\right) .^{51}$ Thus, the anticoagulant effects of SGAT can be explained by inhibition of fibrin formation, which is associated with intrinsic pathway inhibition.

Compared with the positive control, the TT of SGAT was lower than that of heparin. Indeed, the use of $7 \mu \mathrm{g} \mathrm{mL} \mathrm{m}^{-1}$ of heparin prolonged the coagulation time by more than $60 \mathrm{~s}$. Following this, the TT assay of SGAT reported a clotting time of $20.86 \mathrm{IU} \mathrm{mg}^{-1}$ at $50 \mu \mathrm{g} \mathrm{mL}^{-1}$ (Table 3).

The PT test is used to characterize extrinsic coagulation factors. An abnormal or prolonged PT generally designates a deficiency in one or more factors in the extrinsic or common pathway of blood coagulation. This deficiency may be caused by vitamin $\mathrm{K}$ deficiency, hereditary coagulation disorders, liver disease or drug administration.

The anticoagulant activity of SGAT was also evaluated by its ability to extend the prothrombin time (PT). As shown in Fig. 3C, SGAT prolonged the anticoagulant activity in

Table 3 Anticoagulant activity of sulfated glycosaminoglycans purified from Atlantic bluefin tuna skin (SGAT) ${ }^{a}$

\begin{tabular}{|c|c|c|c|c|}
\hline & \multicolumn{2}{|l|}{ aPTT } & \multicolumn{2}{|l|}{ TT } \\
\hline & $\mu \mathrm{g} \mathrm{mL}^{-1}$ & $\mathrm{IU} \mathrm{mg}^{-1}$ & $\mu \mathrm{g} \mathrm{mL}^{-1}$ & $\mathrm{IU} \mathrm{mg}^{-1}$ \\
\hline \multirow[t]{5}{*}{ SGAT } & 25 & 14.39 & 6.25 & 10.8 \\
\hline & 50 & 17.57 & 12.5 & 13 \\
\hline & 100 & 19.88 & 25 & 18.1 \\
\hline & 200 & 27.79 & 50 & 20.86 \\
\hline & 500 & 44.5 & - & - \\
\hline
\end{tabular}

${ }^{a}$ The activity was expressed as international units per $\mathrm{mg}$ using a parallel standard curve based on the International Heparin Standard. a concentration-dependent manner. Furthermore, SGAT showed significantly prolonged PT about 1.28 fold greater than that of the control at a concentration of $500 \mu \mathrm{g} \mathrm{mL} \mathrm{m}^{-1}$. Indeed, SGAT showed a more prolonged PT than that previously described for sulfated GAGs from grey triggerfish (1.1 fold at the same concentration); however, the PT was shorter than that previously described for sulfated GAGs from smooth hound (1.55 fold at $\left.500 \mu \mathrm{g} \mathrm{mL}{ }^{-1}\right) .{ }^{20}$ These results indicate that SGAT has important anticoagulant activity in the extrinsic pathway of blood clots. In this assay, it is impossible to evaluate the PT of heparin because the reagent used (neoplastin) contains a heparin-neutralizing agent.

To conclude, these results suggest that the purified CS/DS recovered from Atlantic bluefin tuna skin may be utilized as an anticoagulant agent both in the inhibition of the intrinsic and extrinsic or common blood coagulation pathways, probably with fewer bleeding side effects than heparin. It has been reported that there is a relationship between the anticoagulant activity and structure of GAGs, such as the degree of sulfation, sulfation position, molecular weight, type of sugar, glycosidic branching and three-dimensional structure of the sulfated polysaccharide..$^{52}$ In this context, Pereira et al. ${ }^{53}$ reported that the disaccharide unit of GAGs monosulfated at the 4-position (Di4S) and/or disulfated at the 2- and 4-positions (Di2,4diS) is necessary for thrombin inhibition by activating heparin cofactor II and antithrombin, respectively. This is in line with the results of our SAX-HPLC analysis.

3.7.2. Hemolytic activity of SGAT. The clinical use of heparin leads to undesirable effects such as hemorrhagic complications due to its capacity to interfere with the hemostatic balance. Thus, it is important to investigate the effects of SGAT on haemostasia (data not shown). The hemolytic activity of SGAT was tested on bovine erythrocytes. Several sample concentrations were tested (25 to $500 \mu \mathrm{g} \mathrm{mL} \mathrm{m}^{-1}$ ). For all concentrations, no hemolysis was observed. These results show that SGAT will be non-toxic even if used at high concentrations.

3.7.3. Angiotensin-I-converting enzyme inhibitory activity. The inhibition of angiotensin I-converting enzyme (ACE) using dietary anti-hypertensive agents is a potentially important strategy to manage hypertension. In this regard, recent research has demonstrated that the ACE inhibition can be considered as a useful therapeutic approach to treat high blood pressure. Because synthetic ACE inhibitors may be a source of adverse side effects, bioactive molecules with antihypertensive activity are receiving much interest because of their beneficial effects in cardiovascular diseases.

The purified SGAT was evaluated in terms of its ACEinhibitory activity. As shown in Fig. 4, SGAT exhibited concentration-dependent ACE inhibitory activity. SGAT was also noted to exhibit strong ACE-inhibitory activity, with the highest levels $(70.81 \%)$ being observed at a concentration of $0.8 \mathrm{mg}$ $\mathrm{mL}^{-1}$. The $\mathrm{IC}_{50}$ value, defined as the concentration of inhibitor required to prevent $50 \%$ of ACE activity, was calculated to be $0.25 \mathrm{mg} \mathrm{mL}{ }^{-1}$. This $\mathrm{IC}_{50}$ value is higher than the $\mathrm{IC}_{50}$ of sulfated polysaccharides from grey triggerfish and smooth hound. ${ }^{18}$

However, Tan and Gan ${ }^{54}$ demonstrated that the ACE inhibitory activity of polysaccharides may be due to the presence of 


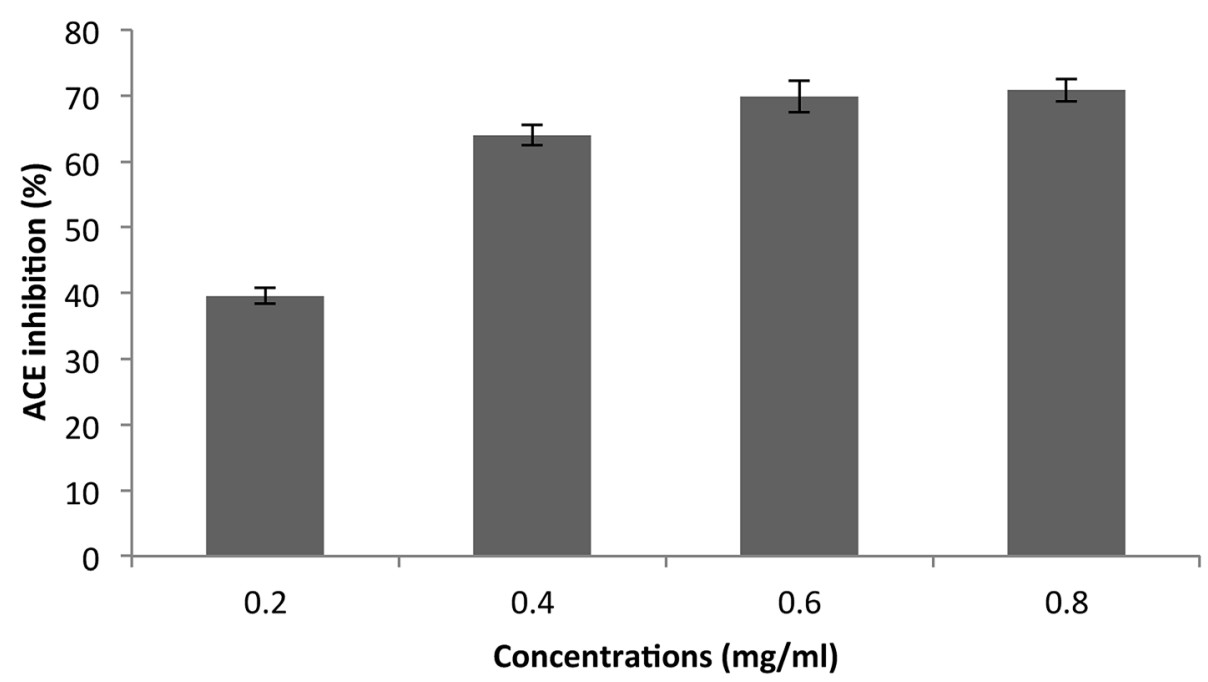

Fig. 4 Angiotensin I-converting enzyme inhibitory activity of SGAT. The values are the means of three replications \pm SD.

uronic acid, which can be ionized. This process creates an acidic environment, which is not suitable for ACE because its optimum $\mathrm{pH}$ is 8.3. Under acidic conditions, ACE would be denatured and therefore lose its activity.

\section{Conclusion}

CS/DS were extracted and purified for the first time from the skin of Atlantic bluefin tuna, and their structural characterization and properties are described in the present study. Furthermore, on the basis of the collected data, CS/DS from tuna skin showed high percentages of monosulfated and disulfated disaccharides produced by chondroitinase $\mathrm{ABC}$ and AC. SGAT had effective in vitro anticoagulant activity; it prolonged blood clotting time based on activated partial thromboplastin time (aPTT), thrombin time (TT) and prothrombin time (PT) tests and exhibited strong ACE-inhibitory activity. Finally, these new CS/DS are potentially useful for pharmacological applications.

\section{Conflicts of interest}

There are no conflicts to declare.

\section{Acknowledgements}

This work was funded by the Tunisian Ministry of Higher Education and Scientific Research.

\section{References}

1 U. Lindahl, Heparin: Chemical and Biological Properties, Clinical Applications, Edward Arnold, London, 1989, vol. 159.

2 L. Kjellen and U. Lindahl, Annu. Rev. Biochem., 1991, 60, 443. 3 V. H. Pomin, Carbohydr. Res., 2015, 413, 41-50.

4 N. Sayari, R. Balti, M. Ben Mansour, I. Ben Amor, I. Graiet, J. Gargouri and A. Bougatef, Biomed. Pharmacother., 2016, 80, 322-330.
5 F. Krichen, W. Karoud, A. Sila, B. E. Abdelmalek, R. Ghorbel, S. Ellouz-Chaabouni and A. Bougatef, Int. J. Biol. Macromol., 2015, 75, 283-289.

6 F. Maccari, F. Galeotti and N. Volpi, Carbohydr. Polym., 2015, 129, 143-147.

7 F. Maccari, F. Ferrarini and N. Volpi, Carbohydr. Res., 2010, 345, 1575-1580.

8 T. Zhao, Y. Zhou, G. Mao, Y. Zou, J. Zhao, S. Bai, L. Yang and X. Wu, J. Sci. Food Agric., 2013, 93, 1633-1640.

9 M. Ben Mansour, H. Majdoub, I. Bataille, M. S. Roudesli, M. Hassine, N. Ajzenberg, F. Chaubet and R. M. Maaroufi, Thromb. Res., 2009a, 123, 671-678.

10 K. Sugahara, S. Nadanaka, K. Takeda and T. Kojima, Eur. J. Biochem., 1996, 239, 871-880.

11 H. Bougatef, F. Krichen, F. Capitani, I. Ben Amor, F. Maccari, V. Mantovani, F. Galeotti, N. Volpi, A. Bougatef and A. Sila, Carbohydr. Polym., 2018, 196, 272-278.

12 F. Krichen, N. Volpi, A. Sila, F. Maccari, V. Mantovani, F. Galeotti, S. Ellouz-Chaabouni and A. Bougatef, Int. J. Biol. Macromol., 2017, 95, 32-39.

13 M. Ben Mansour, M. Dhahri, I. Bertholon, V. Ollivier, I. Bataille, N. Ajzenberg, M. Hassine, M. Jandrot-Perrus, F. Chaubet and R. M. Maaroufi, Thromb. Res., 2009b, 123, 887-894.

14 T. Tsegenidis, Comp. Biochem. Physiol. B, 1992, 103, 275-279. 15 N. Volpi, J. Pharm. Sci., 2007, 96, 3168-3180.

16 N. Volpi, J. Pharm. Pharmacol., 2009b, 61, 1271-1280.

17 C. C. Chatziioannidis, N. K. Karamanos, S. T. Anagnostides and T. Tsegenidis, Biochimie, 1999a, 81, 187-196.

18 F. Krichen, W. Karaoud, N. Sayari, A. Sila, F. Kallel, S. EllouzChaabouni and A. Bougatef, J. Polym. Environ., 2016, 24(2), 166-175.

19 S. F. Chavante, A. S. Brito, M. Lima, E. Yates, H. Nader, M. Guerrini, G. Torri and A. Bisio, Carbohydr. Res., 2014, 390, 59-66.

20 F. Krichen, Z. Ghlissi, I. Ben Amor, N. Sayari, R. Kallel, J. Gargouri, Z. Sahnoun, T. Boudawara, S. Ellouz- 
Chaabouni and A. Bougatef, Exp. Toxicol. Pathol., 2017b, 69, 45-53.

21 A. S. Brito, D. S. Arimatéia, L. R. Souza, M. A. Lima, V. O. Santos, V. P. Medeiros, C. V. Ferreira, G. Z. Justo, E. L. Leite, G. P. Andrade, F. W. Oliveira, H. B. Nader and S. F. Chavante, Bioorg. Med. Chem., 2008, 16, 9588-9595.

22 J. A. Marcum, J. B. McKenney, S. J. Galli, R. W. Jackman and R. D. Rosenberg, Am. J. Physiol., 1986, 250(5), H879-H888.

23 K. Majumder and J. Wu, J. Agric. Food Chem., 2009, 57, 471477.

24 L. Vercruysse, J. V. Camp and G. Smagghe, J. Agric. Food Chem., 2005, 53, 8106-8115.

25 C. P. Alderman, Ann. Pharmacother., 1996, 30, 55-61.

26 M. Cesaretti, E. Luppi, F. Maccari and N. Volpi, Carbohydr. Polym., 2003, 54, 59-61.

27 M. Dubois, K. A. Gilles, J. K. Hamilton, P. A. Rebers and F. Smith, Anal. Chem., 1956, 28, 350-356.

28 Y. Wegrowski and F. X. Maquart, Methods Mol. Biol., 2001, 171, 175-179.

29 R. E. Edens, A. al-Hakim, J. M. Weiler, D. G. Rethwisch, J. Fareed and R. J. Linhardt, J. Pharm. Sci., 1982, 81, 823-827.

30 D. Buzzega, F. Maccari and N. Volpi, J. Pharm. Biomed. Anal., 2010, 51, 969-972.

31 M. Dathe, M. Schumann, T. Wieprecht, A. Winkler, M. Beyermann, E. Krause, K. Matsuzaki, O. Murase and M. Bienert, Biochemistry, 1996, 35, 12612-12622.

32 Y. Nakamura, N. Yamamoto, K. Sakai, A. Okubo, S. Yamazaki and T. Takano, J. Dairy Sci., 1995, 78, 777.

33 B. E. Abdelmalek, A. Sila, F. Krichen, W. Karoud, O. Martinez-Alvarez, S. Ellouz-Chaabouni, M. A. Ayadi and A. Bougatef, Int. J. Biol. Macromol., 2015, 72, 1143-1151.

34 B. W. SSouza, M. A. Cerqueira, A. I. Bourbon, A. C. Pinheiro, J. T. Martins, J. A. Teixeira, M. A. Coimbra and A. A. Vicente, Food Hydrocolloids, 2012, 27, 287-292.

35 M. Ben Mansour, M. Dhahri, M. Hassine, N. Ajzenberg, L. Venisse, V. Ollivier, F. Chaubet, M. Jandrot-Perrus and R. M. Maaroufi, Comp. Biochem. Physiol. B, 2010, 156, 206215.

36 M. E. R. Duarte, M. A. Cardoso, M. D. Noseda and A. S. Cerezo, Carbohydr. Res., 2003, 333, 281-293.
37 D. Santhiya, S. Subramanian and K. A. Natarajan, J. Colloid Interface Sci., 2002, 256, 237-248.

38 S. S. Bandyopadhyay, M. H. Navid, T. Ghosh, P. Schnitzler and B. Ray, Phytochemistry, 2011, 72, 276-283.

39 Y. Kariya, S. Watabe, K. Hashimoto and K. Yoshida, J. Biol. Chem., 1990, 265, 5081-5085.

40 J. Mou, Q. Li, X. Qi and J. Yang, Carbohydr. Polym., 2018, 185, 41-47.

41 G. V. Coppa, F. Maccari, L. Zampini, L. Santoro, T. Galeazzi, O. Gabrielli and N. Volpi, Food Chem., 2012, 134, 195-199.

42 K. A. Jandik, K. Gu and R. J. Linhardt, Glycobiology, 1994, 4, 289-296.

43 T. Hashiguchi, T. Kobayashi, D. Fongmoon, A. K. Shetty, S. Mizumoto, N. Miyamoto, T. Nakamura, S. Yamada and K. Sugahara, Biochim. Biophys. Acta, 2011, 1810, 406-413.

44 C. Malavaki, S. Mizumoto, N. Karamanos and K. Sugahara, Connect. Tissue Res., 2008, 49, 133-139.

45 R. Branislav, M. Marijana and S. Slobodan, World J. Microbiol. Biotechnol., 2008, 24, 1239-1242.

46 E. R. Carbonero, F. R. Smiderle, A. H. P. Gracher, C. G. Mellinger, G. Torri and T. Ahti, Carbohydr. Polym., 2006, 63, 13-18.

47 J. A. G. Rodrigues, I. N. L. Queiroz, A. L. G. Quindere, B. C. Vairo, P. A. S. Mourao and N. M. B. Benevides, Cienc. Rural, 2011, 41, 634-639.

48 J. Yang, Y. Wang, T. Jiang and Z. Lv, Int. J. Biol. Macromol., 2015, 72, 911-918.

49 R. Saravanan and A. Shanmugam, Carbohydr. Polym., 2011, 86, 1082-1084.

50 L. Ye, L. Xu and J. Li, Carbohydr. Polym., 2012, 87(3), 20522057.

51 W. Cai, H. Xu, L. Xie, J. Sun, T. Sun, X. Wu and Q. Fu, Carbohydr. Polym., 2016, 140, 308-313.

52 S. T. Olson, I. Bjork and S. C. Bock, Trends Cardiovasc. Med., 2002, 12, 198-205.

53 M. S. Pereira, F. R. Melo and P. A. S. Mourao, Glycobiology, 2002, 12, 573-580.

54 H. F. Tan and C. Y. Gan, Int. J. Biol. Macromol., 2016, 85, 487496. 